\title{
$\alpha$-lipoic acid attenuates spatial learning and memory impairment induced by hepatectomy
}

\author{
YUAN ZHANG $^{1}$, YUN-LUO LV ${ }^{1}$, YAN-NA SI ${ }^{1}$, JIANWEI ZHOU ${ }^{2}$, YANNING QIAN ${ }^{3}$ and HONG-GUANG BAO ${ }^{1}$ \\ ${ }^{1}$ Department of Anesthesiology, Nanjing First Hospital, Nanjing Medical University, Nanjing, Jiangsu 210006; \\ ${ }^{2}$ Deparment of Molecular Cell Biology and Toxicology, School of Public Health, Nanjing Medical University, \\ Nanjing, Jiangsu 211166; ${ }^{3}$ Department of Anesthesiology, First Affiliated Hospital, Nanjing Medical University, \\ Nanjing, Jiangsu 210029, P.R. China
}

Received December 06, 2017; Accepted November 19, 2018

DOI: $10.3892 /$ etm.2019.7202

\begin{abstract}
The aim of the present study was to compare the effects of $\alpha$-lipoic acid (ALA) on postoperative cognitive dysfunction (POCD) between wild type (WT) and leptin receptor-deficient $(\mathrm{db} / \mathrm{db})$ mice and to elucidate the underlying mechanism of treatment with ALA. The present study compared the effects of ALA on spatial learning and memory of WT and $\mathrm{db} / \mathrm{db}$ mice using a Morris water maze following hepatectomy. The expression levels of proteins, including cyclin-dependent kinase $5(\mathrm{Cdk} 5)$, tau, phosphorylated tau and amyloid $\beta(\mathrm{A} \beta)$ were measured in the hippocampus. Surgery impaired postoperative cognitive function in both WT and db/db mice. Furthermore, the expression levels of Cdk5 and $\mathrm{A} \beta$, and the phosphorylation of tau in the hippocampus increased after the surgery in both WT and $\mathrm{db} / \mathrm{db}$ mice. The ultrastructure of hippocampal neurons and synapses was analyzed by transmission electron microscopy and the results revealed that surgery damaged the structure of neurons and synapses in both WT and db/db mice. Treatment with ALA protected the postoperative cognitive function and the structure of hippocampal neurons and synapses, and prevented the increase in protein expression levels of Cdk5 and $A \beta$, and the phosphorylation of tau in the hippocampus of WT but not $\mathrm{db} / \mathrm{db}$ mice. The results of the present study suggest that ALA may be used for the treatment of POCD. The molecular mechanisms underlying the activity of ALA require further investigation.
\end{abstract}

\section{Introduction}

Postoperative cognitive dysfunction (POCD) is a common postsurgical complication of the central nervous system. POCD occurs at an early stage of postoperative care and increases

Correspondence to: Dr Hong-Guang Bao, Department of Anesthesiology, Nanjing First Hospital, Nanjing Medical University, 68 Changle Road, Nanjing, Jiangsu 210006, P.R. China

E-mail: tracy_kelvin@njmu.edu.cn

Key words: $\alpha$-lipoic acid, postoperative spatial learning and memory impairment, cyclin-dependent kinase 5, tau, amyloid $\beta$, leptin the rates of perioperative mortality (1). The pathogenesis and prevention of POCD have been extensively studied. The mechanism underlying the behavioral alterations following surgery in animal models may be associated with the dysfunction of neurons and synapses (2-6). It has been hypothesized that the production and aggregation of amyloid $\beta(\mathrm{A} \beta)$, and the abnormal hyperphosphorylation and aggregation of tau protein may be involved in the pathogenesis of POCD (7). A $\beta$ may alter the mitochondrial morphology and affect the steady state of calcium in the neurons (8). The hyperphosphorylation and aggregation of tau protein serves an important role in neurodegeneration (9).

The neuroprotective effect of $\alpha$-lipoic acid (ALA) has been previously studied (10) and it was demonstrated that ALA promotes the secretion of leptin from adipocytes (11). A number of studies reported that leptin induces a protective effect on the cognitive function (12-15). Leptin regulates glucose and fat metabolism, and the leptin receptor activates brain-derived neurotrophic factor to regulate the synaptic plasticity and promote neural differentiation $(12,13)$. Furthermore, leptin reduces the hyperphosphorylation of tau protein (14-16).

There are no effective treatment methods for patients with POCD and the underlying mechanism of this conditions remains to be elucidated. To the best of the authors' knowledge, the current study is the first to investigate the effect of ALA on POCD and the underlying mechanism of action. The present study aimed to investigate the effect of ALA on POCD induced by hepatectomy in wild type (WT) and leptin receptor-deficient $(\mathrm{db} / \mathrm{db})$ mice. Protein expression levels of $\mathrm{Cdk} 5$, phosphorylated tau (p-tau) and $A \beta$ were determined by western blotting, and the ultrastructure of hippocampal neurons and synapses was analyzed by transmission electron microscopy.

\section{Materials and methods}

Experimental animals. A total of $60 \mathrm{WT}$ C57BL/6 mice and $60 \mathrm{db} / \mathrm{db}$ C57BL/6 mice (all male; weight, 20-25 g; age, 14 weeks) were obtained from Beijing Vital River Laboratory Animal Technology Co., Ltd. (Beijing, China). Mice were housed with a $12 \mathrm{~h} \mathrm{light/dark}$ cycle at a temperature of $24 \pm 1^{\circ} \mathrm{C}$ with access to food and water ad libitum. ALA (60 mg/kg; Wyeth Pharmaceutical Co., Ltd., Suzhou, China) or 
$1 \%$ dimethyl sulfoxide in corn oil (vehicle) was administrated orally daily. At the end of the experiment, the animals were euthanized by intraperitoneal administration of a lethal dose of sodium pentobarbital $(150 \mathrm{mg} / \mathrm{kg})$. The present study was approved by the Institution Animal Care and Use Committee of Nanjing First Hospital (Nanjing, China).

Animal grouping. C57BL/6 WT and db/db mice were divided randomly into three groups each, including the control, surgery and ALA + surgery groups (20 mice/group). Control group mice received anesthesia only, the surgery group received $70 \%$ hepatectomy surgery, and the ALA + surgery group was intragastrically administrated $100 \mathrm{mg} / \mathrm{kg}$ ALA once daily for 12 weeks (17), and subsequently received $70 \%$ hepatectomy surgery. The control and surgery groups received the same volume of vehicle intragastrically (WT, $20 \mu \mathrm{l} / \mathrm{mice}$; db/db, $30 \mu \mathrm{l} / \mathrm{mice})$.

Hepatectomy surgery. Mice were anesthetized by an intraperitoneal injection of $50 \mathrm{mg} / \mathrm{kg}$ sodium pentobarbital $(1 \%$ in saline). Mice were subsequently placed on a warming blanket and rectal temperatures were monitored. A roll of gauze was placed under the right scapula to provide adequate exposure of the liver. The abdomen of the mice was shaved, sterilized and draped. A 1-1.5-cm midline incision was made from the xiphoid inferiorly with micro dissecting scissors. The upper abdomen was opened and the three anterior lobes of the liver were isolated ( $\sim 68 \%$ of the total liver weight), including the right upper lobe, left upper lobe and left lower lobe. Three knots were tied with moistened silk suture at the base of the lobes near the inferior vena cava. The tied lobes were subsequently cut immediately distal to the suture. The abdomen was irrigated with $2 \mathrm{ml}$ of sterile saline $\left(37^{\circ} \mathrm{C}\right)$ to ensure the removal of blood and to decrease contamination. Gentle pressure was applied on the abdomen with sterile gauze to remove residual irrigation. The wound was infiltrated with $0.25 \%$ bupivacaine to relieve pain. The peritoneum and the skin were subsequently closed separately with silk suture. The mice were placed under warming lights while waking up from anesthesia, and subsequently housed individually (18).

Morris water maze (MWM). Cognitive function was assessed using a 5-day MWM test as previously described (19). A round tub was used for the MWM task and filled with water mixed with nontoxic black paint to submerge a platform $1 \mathrm{~cm}$ below the water surface (water temperature, $19-24^{\circ} \mathrm{C}$ ). Visual distal cues were located on the walls. During the first four days, mice were given four trials/day using a random starting location. If mice did not reach the platform within $60 \mathrm{sec}$, they were gently guided to the hidden platform. The latency to reach the platform was recorded from all sessions, and averaged to calculate the escape latency for each day. On day 5, the platform was removed for probe testing. Mice were allowed to swim freely for $60 \mathrm{sec}$. The number of crossings over the platform that had served as the target on days 1-4 was recorded. The time spent in the quadrant where the target platform was located was recorded. Data were collected and analyzed using motion detection software (DigBehv-MM; Shanghai Jiliang Software Technology Co., Ltd., Shanghai, China).
Transmission electron microscopy. Mice were intraperitoneally injected with $1 \%$ pentobarbital sodium $(35 \mathrm{mg} / \mathrm{kg})$ and then fixed on a foam plate. A large U-shaped incision was cut into the chest to expose the heart. The perfused needle was inserted into the apical part with $5 \mathrm{~mm}$ depth and fixed by hemostatic clamp. Heparin saline was infused until liver became pale, followed with $3 \%$ glutaraldehyde perfusion. The brain was removed, and the hippocampus was isolated and post-fixed in $2.5 \%$ glutaraldehyde solution for $2 \mathrm{~h}$ in $4^{\circ} \mathrm{C}$, followed by embedding in the epoxy resin for $12 \mathrm{~h}$ at $45^{\circ} \mathrm{C}$. The embedded tissues were cut into 50-70-nm-thick sections and mounted on 150 mesh copper grids. Following staining with uranyl acetate and lead citrate for $12 \mathrm{~h}$ in $4^{\circ} \mathrm{C}$, the specimens were observed under a transmission electron microscope (HITACHI-7650; Hitachi, Ltd., Tokyo, Japan).

Western blotting. The hippocampal tissue was homogenized with Tris- $\mathrm{HCl}(\mathrm{pH} \mathrm{7.4;50} \mathrm{mM),} \mathrm{1 \%} \mathrm{Triton} \mathrm{X-100,} \mathrm{0.2 \%}$ sodium deoxycholate, $0.2 \% \mathrm{SDS}$ and $1 \mathrm{mM}$ EDTA (Beyotime Institute of Biotechnology, Shanghai, China), and centrifuged at $8,000 \mathrm{x} \mathrm{g}$ for $20 \mathrm{~min}$ at $4^{\circ} \mathrm{C}$. The protein concentration from each mouse was tested using the bicinchoninic acid method. Protein (40 $\mu \mathrm{g} /$ lane) was separated by SDS-PAGE (12\% gel) and transferred to a nitrocellulose membrane (Hybond ${ }^{\circledR}$ ECL $^{\mathrm{TM}}$; GE Healthcare, Chicago, IL, USA). Membranes were blocked with $5 \%$ milk and $0.1 \%$ Tween-20 in PBS for $1 \mathrm{~h}$ at room temperature. Membranes were then incubated with primary antibodies against Cdk5 (cat. no. ab21249), tau (cat. no. ab80579), $\beta$-actin (cat. no. ab8227; all 1:1,000), A $\beta$ (cat. no. ab10148; 1:7,000; all Abcam, Cambridge, MA, USA) and p-tau (cat. no. 44-750G; 1:1,000; Thermo Fisher Scientific, Inc., Waltham, MA, USA) overnight at $4^{\circ} \mathrm{C}$. After washing with tris buffered saline with Tween-20 (TBST) three times, membranes were incubated with anti-rabbit secondary antibodies (cat. no. ab6721; 1:2,000; Abcam) for $2 \mathrm{~h}$ at room temperature. Between steps, blots were washed with TBST. Immunodetection was performed using a LumiGLO chemiluminescence kit (Amersham; GE Healthcare). Bands were analyzed by Quantity One software (version 4.6.2; Bio-Rad Laboratories, Inc., Hercules, CA, USA).

Statistical analysis. All data are presented as the mean \pm standard deviation. Data were analyzed using SPSS software (version 17.0; SPSS, Inc., Chicago, IL, USA) or GraphPad Prism (version 5.0; GraphPad Software, Inc., La Jolla, CA, USA). Two-way analysis of variance (ANOVA) was used to analyze the spatial memory data obtained from the MWM test. The levels of protein and gene expression were analyzed using one-way ANOVA. ANOVA analyses were followed by the Bonferroni post-hoc test. $\mathrm{P}<0.05$ was considered to indicate a statistically significant difference.

\section{Results}

ALA rescues the impaired cognitive function in WT but not $\mathrm{db} / \mathrm{db}$ mice. To study cognitive function, MWM was used to examine spatial learning and memory in both WT and $\mathrm{db} / \mathrm{db}$ mice with or without treatment with ALA. Among the WT

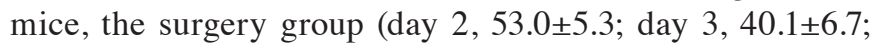
day $4,33.8 \pm 4.1$ ) exhibited significantly longer latency to find the platform compared with the control group (day 2, 27.7 \pm 3.7 ; 
A
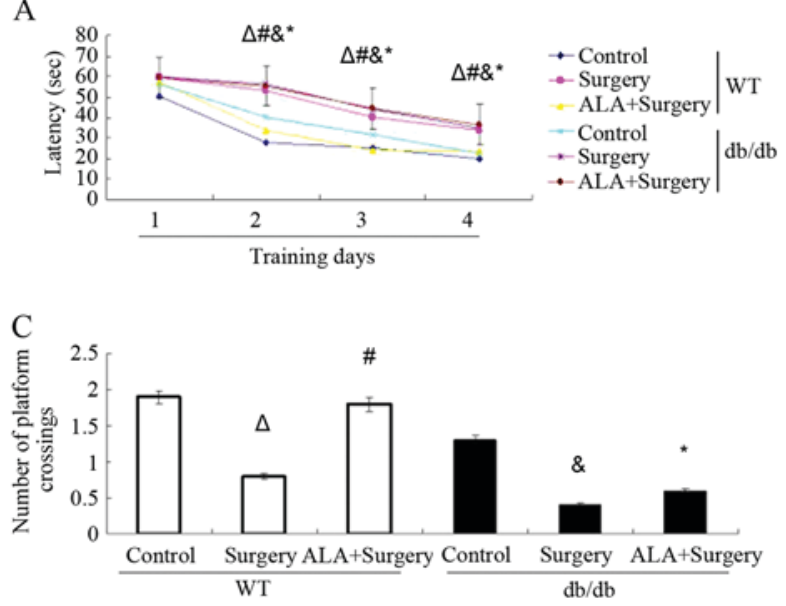

B

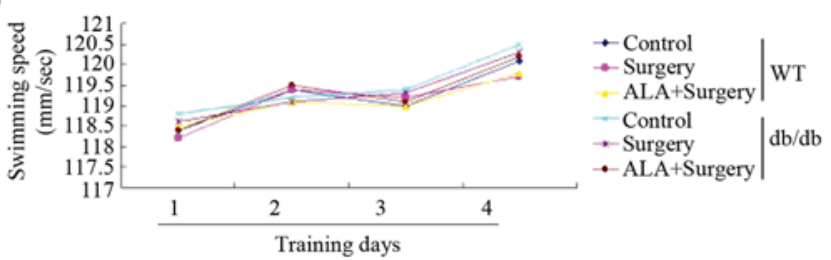

$\mathrm{D}$

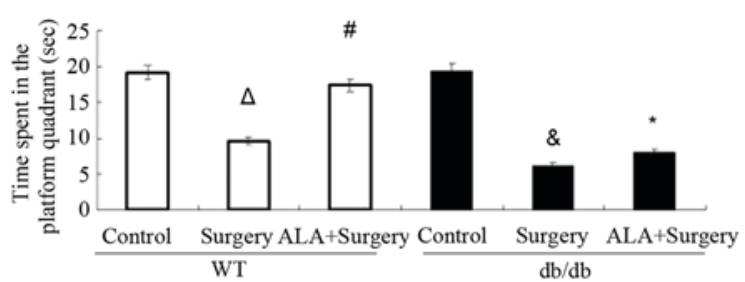

Figure 1. Effects of pretreatment with ALA on surgery-induced behavioral deficits measured using the Morris water maze. (A) The latency to reach the target platform during training days 1-4. (B) The swimming speed during training days 1-4. (C) The time spent in the target quadrant on the testing day (day 5). (D) The number of crossings over the original target platform on the testing day (day 5). Data are represented as the mean \pm standard deviation ( $\mathrm{n}=20$ /group). ${ }^{\Delta} \mathrm{P}<0.01$ compared with the WT control group; ${ }^{*} \mathrm{P}<0.05$ compared with the WT surgery group; ${ }^{\text {}} \mathrm{P}<0.01$ compared with the db/db control group; ${ }^{*} \mathrm{P}<0.01$ compared with the WT ALA + surgery group. ALA, $\alpha$-lipoic acid; WT, wild type; db/db, leptin receptor-deficient mice.

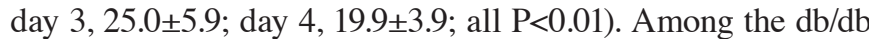
mice, the surgery group (day $2,56.3 \pm 2.1$; day $3,43.8 \pm 3.0$; day 4 , $35.0 \pm 1.5$ ) exhibited significantly longer latency to find the platform compared with the control group (day 2, 40.3 \pm 6.9 ; day 3 , 31.8 \pm 6.3 ; day 4, 22.3 $\pm 3.9 ; \mathrm{P}<0.01)$. The $\mathrm{db} / \mathrm{db}$ ALA + surgery group (day $2,55.4 \pm 2.2$; day $3,44.5 \pm 4.8$; day 4 : 36.7 \pm 4.3 ) did not exhibit altered latency compared with the surgery only group (P>0.05; Fig. 1A). However, the db/db ALA + surgery group exhibited significantly longer latency compared with the WT ALA + surgery group $(\mathrm{P}<0.01$; Fig. 1A). These results suggest that hepatectomy surgery prolonged the escape latency during the MWM test in both WT and db/db mice. Treatment with ALA alleviated the effect induced by surgery in WT mice but not in $\mathrm{db} / \mathrm{db}$ mice. The swimming speed was recorded during the leaning sessions in the MWM test. The results revealed that there were no differences in the swimming speed among the control, surgery and ALA + surgery groups in WT and $\mathrm{db} / \mathrm{db}$ mice (Fig. 1B).

On probe testing day, WT mice in the surgery group crossed the target quadrant fewer times $(0.8 \pm 0.5$ vs. $1.9 \pm 0.8$; $\mathrm{P}<0.01)$ and spent less time in the platform quadrant $(9.6 \pm 7.7$ vs. $19.2 \pm 6.3 ; \mathrm{P}<0.01)$ compared with the control group. Treatment with ALA increased the time spent in the platform quadrant $(17.4 \pm 8.0$ vs. $9.6 \pm 7.7 ; \mathrm{P}<0.05)$ and crossing number $(1.8 \pm 0.6$ vs. $0.8 \pm 0.5 ; \mathrm{P}<0.05)$ in the target quadrant compared with the surgery group. Among the $\mathrm{db} / \mathrm{db}$ mice, the surgery group crossed the target quadrant fewer times $(0.4 \pm 0.3$ vs. $1.3 \pm 0.9 ; \mathrm{P}<0.01)$ and spent less time in the platform quadrant $(6.2 \pm 3.0$ vs. $19.5 \pm 6.9 ; \mathrm{P}<0.01)$ compared with the control group. However, the crossing number $(0.6 \pm 0.4$ vs. $0.4 \pm 0.3$; $\mathrm{P}>0.05)$ and time spent in the platform quadrant $(8.0 \pm 5.4$ vs. $6.2 \pm 3.0 ; \mathrm{P}>0.05)$ remained unaltered following administration of ALA compared with the surgery group among the $\mathrm{db} / \mathrm{db}$ mice (Fig. 1C and D). The ALA + surgery group $\mathrm{db} / \mathrm{db}$ mice spent less time in the platform quadrant $(8.0 \pm 3.1$ vs. $17.4 \pm 8.0 ; \mathrm{P}<0.01)$ and crossed the platform fewer times $(0.6 \pm 0.3$ vs. $1.8 \pm 0.6 ; \mathrm{P}<0.05)$ compared to ALA + surgery group WT mice $(\mathrm{P}<0.01$; Fig. $1 \mathrm{C}$ and $\mathrm{D})$.
Hepatectomy increases $C d k 5, p$-tau and $A \beta$ protein levels in both WT and db/db mice, and this effect is reversed following treatment with ALA among WT mice but not db/db mice. Western blotting was used to assess the expression levels of cognitive function-associated proteins Cdk5 and $A \beta$ and the phosphorylation levels of tau. Among the WT mice, surgery significantly increased the protein expression of $\mathrm{Cdk} 5$ and $\mathrm{A} \beta$ and the $\mathrm{p} /$ total $(\mathrm{t})$ tau ratio in the hippocampus compared with the control group $(\mathrm{P}<0.01)$. Administration pf ALA following surgery significantly reduced the protein expression of $\mathrm{Cdk} 5$ and $\mathrm{A} \beta$, and the $\mathrm{p} / \mathrm{t}$ tau ratio in the hippocampus compared with the surgery group ( $\mathrm{P}<0.05$; Fig. 2).

Among the $\mathrm{db} / \mathrm{db}$ mice, surgery significantly increased the protein expression of $\mathrm{Cdk} 5$ and $\mathrm{A} \beta$ and the $\mathrm{p} /$ total $(\mathrm{t})$ tau ratio in the hippocampus compared with the control group $(\mathrm{P}<0.01)$. However, treatment with ALA did not alter the protein expression of $\mathrm{Cdk} 5$ and $A \beta$ or the $\mathrm{p} / \mathrm{t}$ tau ratio compared with the surgery group. The $\mathrm{db} / \mathrm{db}$ ALA + surgery group mice exhibited significantly increased expression of Cdk5 and $A \beta$, and $p / t$ tau ratio in the hippocampus compared with the WT ALA + surgery group in mice $(\mathrm{P}<0.01$; Fig. 2).

Hepatectomy impairs cellular structure of the hippocampus of WT and db/db mice, and this effect is reversed following treatment with ALA among WT mice but not db/db mice. The ultrastructure of the CA3 hippocampal region was analyzed using transmission electron microscopy (Fig. 3). The images revealed that among the WT mice, surgery (Fig. 3B) impaired the ultrastructure of hippocampal neurons, which exhibited pyknosis, shrinkage of nuclear membrane, broadened perinuclear space and reduced synaptic density. Administration of ALA after surgery (Fig. 3C) improved the neuronal structure in the hippocampus, which appeared similar to the control group (Fig. 3A), exhibiting a smooth nuclear membrane and normal synaptic density and structure.

Among the db/db mice, surgery (Fig. 3E) impaired the ultrastructure of hippocampal neurons and synapses compared with the control group. However, treatment with ALA + surgery 


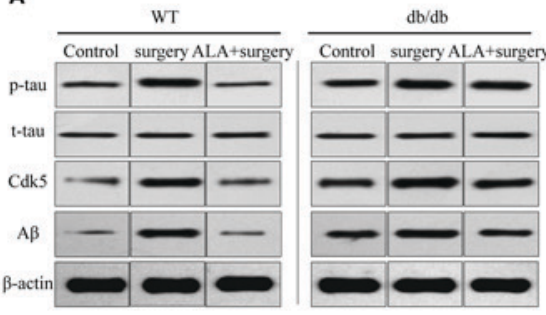

D

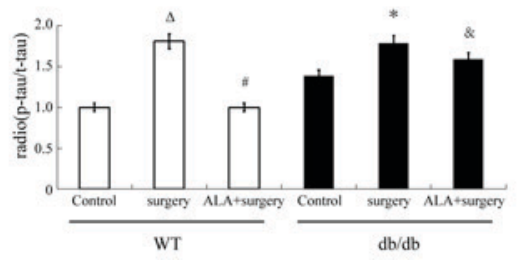

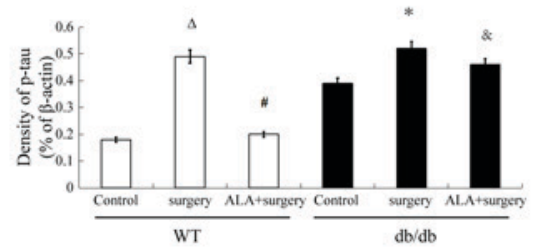

E

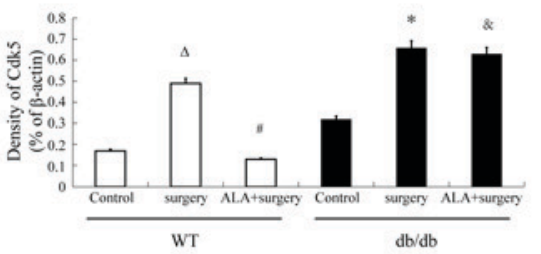

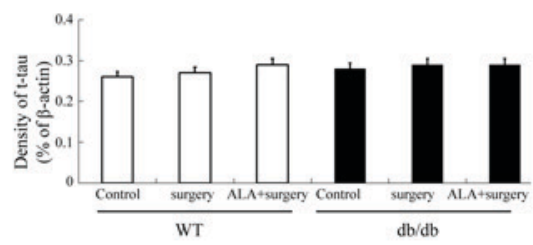

$\mathrm{F}$

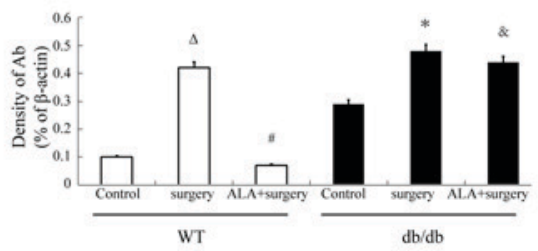

Figure 2. Effects of ALA on surgery-induced alterations in Cdk5 and A $\beta$ protein expression levels and $p / t$ tau ratio in the hippocampus. (A) Representative images of western blotting for the analysis of $\mathrm{p}$-tau, t-tau, $\mathrm{Cdk} 5, \mathrm{~A} \beta$ and $\beta$-actin protein expression. (B) Quantitative analysis of $\mathrm{p}$-tau protein expression relative to $\beta$-actin. (C) Quantitative analysis of t-tau protein expression relative to $\beta$-actin. (D) Quantitative analysis of $\mathrm{p}$-tau to t-tau protein expression ratio. (E) Quantitative analysis of Cdk5 protein expression relative to $\beta$-actin. (F) Quantitative analysis of $A \beta$ protein expression relative to $\beta$-actin. Data are presented as the mean \pm standard deviation ( $\mathrm{n}=5$ /group). ${ }^{\Delta} \mathrm{P}<0.01$ compared with the $\mathrm{WT}$ control group; ${ }^{*} \mathrm{P}<0.05$ compared with the surgery group; ${ }^{\mathrm{P}} \mathrm{P}<0.01 \mathrm{compared}$ with the $\mathrm{db} / \mathrm{db}$ control group; ${ }^{\circledR} \mathrm{P}<0.01$ compared with the WT ALA + surgery group. ALA, $\alpha$-lipoic acid; WT, wild type; db/db, leptin receptor-deficient mice; p, phosphorylated; t, total; Cdk5, cyclin-dependent kinase 5; A $\beta$, amyloid $\beta$.

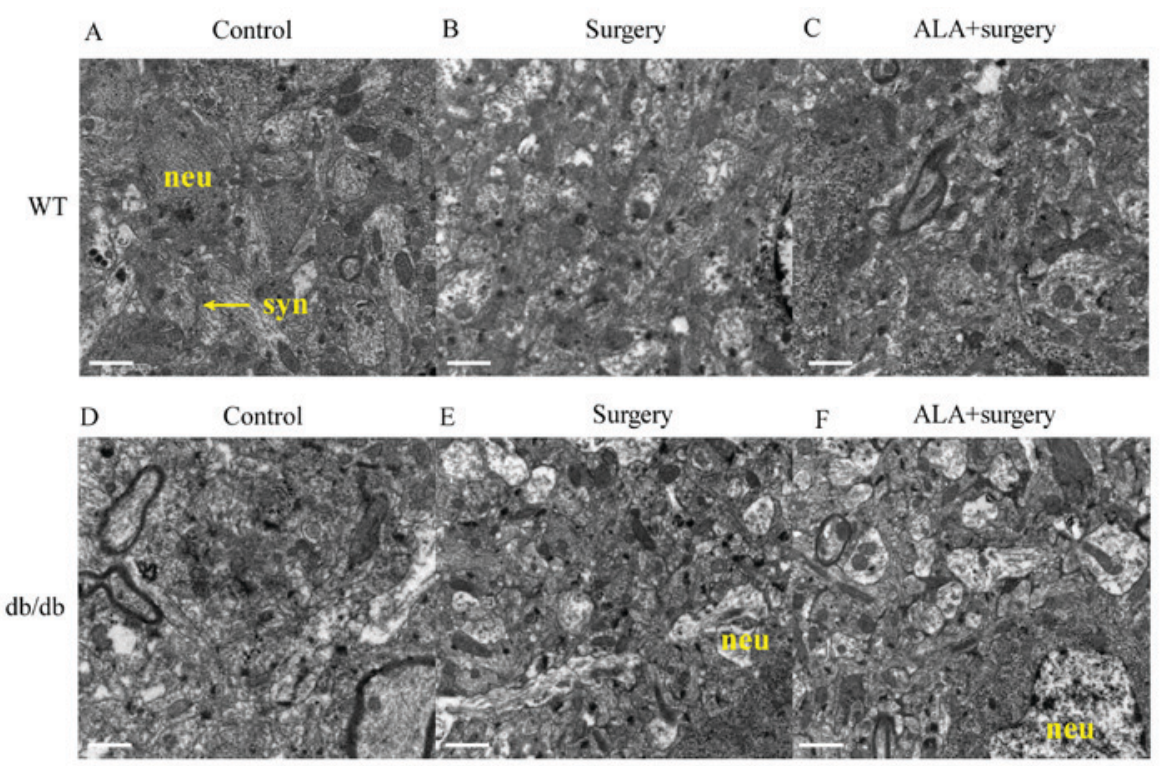

Figure 3. Ultrastructure of neurons and synapses in the hippocampus. The ultrastructure images from electron microscopy presenting the results for (A) WT control group, (B) WT surgery group, (C) WT ALA + surgery group, (D) db/db control group, (E) db/db surgery group, (F) db/db ALA+surgery group. Scale bar $=1 \mu \mathrm{m}$. Neu, neuron; syn, synapse; ALA, $\alpha$-lipoic acid; WT, wild type; db/db, leptin receptor-deficient mice.

(Fig. 3F) did not repair the neuronal structure in the hippocampus, which was different compared with the normal structure of the control group (Fig. 3D).

\section{Discussion}

A previous study reported that ALA can increase the level of leptin in serum through the regulation of Cdk5. Cdk5 serves an important role in neuron differentiation and synaptic plasticity by phosphorylating cytoskeleton proteins and signaling pathway molecules (16). Cdk5 is a serine/threonine protein kinase that forms complexes with p35 or p39 and is essential for neural development and function (20). Downregulation of CDK5 has been demonstrated to mitigate hippocampal degeneration and cognitive dysfunction (16).

The effects of ALA on the cognitive function following hepatectomy and the possible underlying mechanism were studied using $\mathrm{db} / \mathrm{db}$ and WT mice. The results revelated that surgery impaired postoperative cognitive function, increased the hippocampal expression of $\mathrm{Cdk} 5$ and $\mathrm{A} \beta$ proteins and the 
phosphorylation of tau, and damaged the structure of hippocampal neurons and synapses in both WT and $\mathrm{db} / \mathrm{db}$ mice. ALA rescued the cognitive function of WT mice after surgery, as revealed by the results of the MWM learning and test.

In addition, analysis of protein expression in the hippocampus revealed that treatment with ALA decreased the elevated protein expression levels of $\mathrm{Cdk} 5$ and $A \beta$ and the $p / t$ tau ratio in WT mice subjected to surgery. However, this effect was not observed among the $\mathrm{db} / \mathrm{db}$ mice. The ultrastructure of neurons and synapses in the hippocampus was observed in mice following surgery and treatment with ALA. Hepatectomy markedly damaged the structure of neurons and synapses in both $\mathrm{WT}$ and $\mathrm{db} / \mathrm{db}$ mice. However, treatment with ALA repaired the structure of neurons and synapses in WT mice, but not the $\mathrm{db} / \mathrm{db}$ mice. These results suggested that the improvement in cognitive function following administration of ALA may be associated with reduced protein expression levels of $\mathrm{Cdk} 5$ and $\mathrm{A} \beta$, and the phosphorylation level of tau. Furthermore, treatment with ALA enabled maintenance of the normal structure of hippocampal neurons and synapses.

Treatment with ALA did not improve the cognitive function of $\mathrm{db} / \mathrm{db}$ mice following hepatectomy, which may suggest that the leptin signaling pathway may be a potential target of ALA. ALA may regulate the expression of leptin or regulate the biding to leptin receptor to alter the expression levels of Cdk5 and $A \beta$, and the phosphorylation level of tau to maintain the normal structure of hippocampal neurons and synapses. Future studies should investigate the effect of ALA on the leptin signaling pathway and cognitive function in WT mice with hepatectomy.

\section{Acknowledgements}

Not applicable.

\section{Funding}

The current study was supported by grants from the National Natural Science Foundation (grant no. 81873954) and the Medicine Development Project of Nanjing Scientific Committee (grant no. YKK15089).

\section{Availability of data and materials}

All data generated or analyzed during this study are available from the corresponding author on reasonable request.

\section{Authors' contributions}

YZ, HGB conceived the study design. YZ and YLL performed the experiments. YNS, JWZ and YNQ participated in the data analysis and interpretation.

\section{Ethics approval and consent to participate}

\section{Competing interests}

The authors declare that they have no competing interests.

\section{References}

1. Krenk L and Rasmussen LS: Postoperative delirium and postoperative cognitive dysfunction in the elderly-what are the differences? Minerva Anestesiol 77: 742-749, 2011.

2. Hovens IB, Schoemaker RG, van der Zee EA, Absalom AR, Heineman E and van Leeuwen BL: Postoperative cognitive dysfunction: Involvement of neuroinflammation and neuronal functioning. Brain Behav Immun 38: 202-210, 2014.

3. Mézière A, Paillaud E and Plaud B: Anesthesia in the elderly. Presse Med 42: 197-201, 2013 (In French).

4. Miyoshi E, Wietzikoski EC, Bortolanza M, Boschen SL, Canteras NS, Izquierdo I and Da Cunha C: Both the dorsal hippocampus and the dorsolateral striatum are needed for rat navigation in the Morris water maze. Behav Brain Res 226: 171-178, 2012

5. Sawamura S: Postoperative care of the elderly. Nihon Rinsho 71: 1060-1064, 2013 (In Japanese).

6. Wuri G, Wang DX, Zhou Y and Zhu SN: Effects of surgical stress on long-term memory function in mice of different ages. Acta Anaesthesiol Scand 55: 474-485, 2011.

7. Xie Z and Tanzi RE: Alzheimer's disease and post-operative cognitive dysfunction. Exp Gerontol 41: 346-359, 2006.

8. Hovens IB, Schoemaker RG, van der Zee EA, Heineman E, Nyakas $C$ and van Leeuwen BL: Surgery-induced behavioral changes in aged rats. Exp Gerontol 48: 1204-1211, 2013.

9. Gustafson DR: Adiposity and cognitive decline: Underlying mechanisms. J Alzheimers Dis 30 (Suppl 2): S97-S112, 2012.

10. Deng C, Sun Z, Tong G, Yi W, Ma L, Zhao B, Cheng L, Zhang J, Cao $F$ and Yi D: $\alpha$-Lipoic acid reduces infarct size and preserves cardiac function in rat myocardial ischemia/reperfusion injury through activation of PI3K/Akt/Nrf2 pathway. PLoS One 8: e58371, 2013.

11. Kandeil MA, Amin KA, Hassanin KA, Ali KM and Mohammed ET: Role of lipoic acid on insulin resistance and leptin inexperimentally diabetic rats. J Diabetes Complications 25: 31-38, 2011.

12. Gupta A: Leptin as a neuroprotective agent in glaucoma. Med Hypotheses 81: 797-802, 2013.

13. Folch J, Pedrós I, Patraca I, Sureda F, Junyent F, Beas-Zarate C, Verdaguer E, Pallàs M, Auladell C and Camins A: Neuroprotective and anti-ageing role of leptin. J Mol Endocrinol 49: R149-R156, 2012.

14. Irving AJ and Harvey J: Leptin regulation of hippocampal synaptic function in health and disease. Philos Trans R Soc Lond B Biol Sci 369: 20130155, 2014.

15. Sánchez JC, Ospina JP and González MI: Association between leptin and delirium in elderly inpatients. Neuropsychiatr Dis Treat 9: 659-666, 2013.

16. Shukla V, Skuntz S and Pant HC: Deregulated Cdk5 activity is involved in inducing Alzheimer's disease. Arch Med Res 43: 655-662, 2012.

17. Chen WL, Kang CH, Wang SG and Lee HM: $\alpha$-Lipoic acid regulates lipid metabolism through induction of sirtuin 1 (SIRT1) and activation of AMP-activated protein kinase. Diabetologia 55: 1824-1835, 2012.

18. Higgins GM and Anderson RM: Experimental pathology of the liver. I. Restoration of the liver of the white rat following partial surgical removal. Arch Pathol 12: 186-202, 1931.

19. Ku S, Yan F, Wang Y, Sun Y, Yang N and Ye L: The blood-brain barrier penetration and distribution of PEGylated fluorescein-doped magnetic silica nanoparticles in rat brain. Biochem Biophys Res Commun 394: 871-876, 2010.

20. Wilkaniec A, Gąssowska-Dobrowolska M, Strawski M, Adamczyk A and Czapski GA: Inhibition of cyclin-dependent kinase 5 affects early neuroinflammatory signalling in murine model of amyloid beta toxicity. J Neuroinflammation 15: 1, 2018.

Not applicable.

\section{Patient consent for publication}

Not applicable. 